'Escuela de Kinesiología, Facultad de Salud, Universidad Santo Tomás, Chile.

2 Núcleo de Investigación Interdisciplinario en Salud (NIIS), Facultad de Salud, Universidad Santo Tomás, Chile.

aKinesióloga Mg. en Educación.

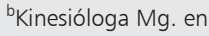
Administración de Empresas (MBA).

'Kinesiólogo Mg. en fisiología clínica del ejercicio.

${ }^{d}$ Kinesiólogo MSc. de la Salud y el Deporte, PhD. en Ciencias de la Actividad Física.

EKinesiólogo MSc. de la Salud y el Deporte, PhD. en Ciencias de la Actividad Física y del Deporte.

Trabajo no recibió financiamiento. Los autores declaran no tener conflictos de interés.

Recibido el 6 de noviembre de 2020, aceptado el 23 de marzo de 2021.

Correspondencia a: Guillermo Méndez-Rebolledo Kinesiólogo MSc. PhD. Avenida Circunvalación \#1855, Talca, Chile. guillermomendezre@ santotomas.cl

\section{Factores asociados a la disminución de funcionalidad del miembro superior en estudiantes universitarios chilenos en pandemia por COVID-19}

\author{
IVONNE JORQUERA-CÁCERES ${ }^{1,2, a}$, CLAUDIA LIRA-JORQUERA ${ }^{1,2, \mathrm{~b}}$, \\ DANY SOBARZO-SOTO ${ }^{1,2, \mathrm{c}}$, EDUARDO GUZMÁN-MUÑOZ ${ }^{1,2, \mathrm{~d}}$, \\ GUILLERMO MÉNDEZ-REBOLLEDO ${ }^{1,2, \mathrm{e}}$
}

\section{Risk factors associated with a lower upper limb functionality among university students}

Background: Upper limb (UL) function is compromised in university students due to prolonged academic activities and excessive use of electronic devices. However, it is unknown whether this relationship was affected by the COVID-19 pandemic situation. Aim: To determine the risk factors associated with reduced UL functionality in university students in a pandemic situation due to COVID-19. Material and Methods: Cross-sectional study that included 621 university students from the northern, central and southern areas of Chile. Physical variables, musculoskeletal, anthropometric and sociodemographic history were evaluated through self-reported questionnaires. Results: The factors with the higher association with a lower UL functionality were pain (Odds ratio $(O R)=17.6 ; 95 \%$ confidence intervals $(C I)=5.2-60 ; p<0.01)$ and injury $(O R=10.4 ; 95 \% C I=3.9-28.3 ; p<.001)$. Also, a low weight $(O R=10.5$; $95 \% C I=2.2-49.6 ; p<0.01)$ and a quarantine lasting for five or more weeks $(O R=9.1 ; 95 \% C I=1,1-77.2 ; p=0.043)$ were considered risk factors. The lower probability was observed in male participants $(O R=0.2 ; 95 \% C I=0.1-0.51$; $p<0.01)$ and in those with a moderate/high muscular condition $(O R=0.3$; 95\% CI $=0.1-0.9 ; p=0.031$. Conclusions: The identification of these modifiable risk factors can help to implement preventive or rehabilitative actions at home or universities.

(Rev Med Chile 2021; 149: 836-845)

Key words: Coronavirus; Disability Evaluation; Pandemics; Students; Upper Extremit.
E 1 nuevo coronavirus SARS-CoV-2 generó que el 30 de enero del año 2020 la OMS declarara que la enfermedad causada por éste, COVID-19, fuera considerada un evento de salud pública Internacional ${ }^{1}$. Los países del continente Latinoamericano, incluyendo Chile, fueron los más afecados ${ }^{2}$, provocando consecuencias sociales y económicas ${ }^{3}$. Entre las diversas estrategias implementadas para mitigar el contagio, se aplicó el aislamiento social preventivo y el confinamiento en casa, y se restringieron los desplazamientos a lugares de actividad y ejercicio físico ${ }^{4,5}$.

Estudios previos a la situación de pandemia por COVID-19, han reportado que los estudiantes universitarios chilenos tienden a mantener conductas sedentarias y un bajo nivel de actividad física ${ }^{6}$, asociado a una mayor probabilidad de desarrollar enfermedades como obesidad, diabetes 
e hipertensión ${ }^{6,7}$, y a una menor percepción de la calidad de vida relacionada con la salud ${ }^{8}$. Esto se suma a que una mayor dedicación a horas de estudio y una alta exposición al uso de pantallas (computador y/o smartphone) lo que promueve la adquisición de estilos de vida no saludables en esta población ${ }^{6,9,10}$. En la actualidad, debido a las restricciones de distanciamiento social impuestas por los gobiernos debido a la pandemia por COVID-19, muchos jóvenes universitarios han debido reemplazar las actividades académicas presenciales por actividades académicas online. Este cambio podría incrementar los factores de riesgo que influyen en la funcionalidad, ya que se ha demostrado que el uso por períodos prolongados y sin descanso de dispositivos electrónicos puede ser perjudicial para la salud ${ }^{10,11}$. En este sentido, se ha propuesto que la evaluación de la funcionalidad del miembro superior puede ser un buen indicador de discapacidad, limitaciones de actividad y restricción de participación ${ }^{12}$. Se ha reportado una asociación negativa entre dolor musculoesquelético cervical-lumbar y pérdida de funcionalidad de miembro superior en actividades de la vida diaria en estudiantes universitarios ${ }^{13}$, como también, altos niveles de tensión en músculos cervicales y trapecio en escolares expuestos a varias horas de uso de computador en sus actividades académicas ${ }^{13-15}$.

Mantener niveles apropiados de actividad física, un adecuado estado nutricional y evitar conductas sedentarias, pueden reducir la mortalidad, la incidencia de enfermedades crónicas, y el deterioro de la funcionalidad, además de otorgar beneficios para la salud en general ${ }^{16-18}$. Sin embargo, no se han reportado investigaciones sobre una posible asociación entre el nivel de funcionalidad del miembro superior y diversos factores sociodemográficos, antropométricos, musculoesqueléticos, y nivel de actividad física en estudiantes universitarios chilenos en situación de pandemia por COVID-19, por lo que se desconocen los principales factores de riesgo relacionados, y cuál de ellos podrían tener una mayor o menor implicancia en la disminución de la funcionalidad del miembro superior. Por lo anterior, el objetivo de este estudio fue determinar los factores de riesgo asociados a una menor funcionalidad del miembro superior en estudiantes universitarios en situación de pandemia por COVID-19.

\section{Materiales y Métodos}

\section{Diseño del estudio}

Estudio descriptivo de corte transversal. La muestra seleccionada comprendió a estudiantes matriculados y vigentes del 2020 en la carrera de kinesiología de una universidad presente en las ciudades de Arica, Iquique, Viña del Mar, Santiago, Talca, Los Ángeles, Osorno y Puerto Montt. Se calcularon 328 participantes a partir de un muestreo aleatorio estratificado por sedes y género, considerando una población de 1.571 individuos, una heterogeneidad de $50 \%$, un margen de error de $5 \%$ y un nivel de confianza de $95 \%$.

Los criterios de inclusión utilizados fueron: contar con matrícula vigente durante al mes de abril del año 2020 y tener $\geq 18$ años. Los criterios de exclusión fueron: estudiantes que al momento de la recolección de datos tenían licencia médica, se encontraran realizando su práctica profesional, o presentaban contraindicaciones para realizar actividad física. Fueron reclutados 621 participantes quienes firmaron un consentimiento informado de forma digital previo a la recolección de los datos. Todos los participantes respondieron la encuesta y cumplieron los criterios anteriormente mencionados. Los datos fueron recolectados mediante un cuestionario de auto-reporte a través de la plataforma online Google forms. El protocolo de esta investigación fue aprobado por el Comité de Ética de la Universidad bajo el código 68-19.

\section{Funcionalidad del miembro superior}

Se utilizó el cuestionario de auto-reporte Discapacidades del Brazo, Hombro y Mano (DASH, del inglés Disabilities of Arm, Shoulder and Hand). Se consideró el módulo discapacidad/síntomas consistente en 30 ítems destinados a medir el impacto de la lesión de miembro superior al realizar tareas funcionales ${ }^{19}$. El cuestionario permite valorar la discapacidad percibida por el participante para realizar diversas actividades, incluidas actividades de la vida diaria y síntomas como el dolor, la rigidez o la pérdida de fuerza ${ }^{19}$. La puntuación total puede oscilar entre 30 y 150 puntos y se transforma en una escala de 0 (mejor puntuación posible) a 100 (peor puntuación posible) ${ }^{19}$. En este estudio la variable fue categorizada como funcionalidad conservada (DASH $<15$ puntos) o disminuida (DASH $\geq 15$ puntos) basado en investigaciones previas $^{20,21}$. 


\section{Variables físicas yantecedentes musculoesqueléticos, antropométricos y sociodemográficos}

El nivel de actividad física se midió a través del Cuestionario Internacional de Actividad Física (IPAQ) versión corta ${ }^{22}$. El indicador de actividad física total fue expresado de manera continua en METs (Metabolic-energy-equivalents) y se dicotomizaron los resultados en baja actividad física cuando el gasto energético fue $\leq 599 \mathrm{METs} / \mathrm{min} /$ semana y moderada-alta actividad física cuando el gasto energético fue $\geq 600 \mathrm{METs} / \mathrm{min} / \mathrm{semana}^{6,22,23}$.

La condición física se midió mediante el cuestionario de auto-reporte de la condición física The International Fitness Scale (IFIS) que evalúa los principales determinantes de la condición física relacionados con la salud ${ }^{24}$. Este cuestionario evalúa 5 dimensiones de la condición física: a) condición física general; b) condición cardiorrespiratoria; c) condición muscular (fuerza); d) velocidad/ agilidad; e) flexibilidad. Los participantes debieron responder como perciben su condición física en cada una de estas dimensiones ${ }^{24}$.

La presencia de antecedentes de dolor y lesión musculoesquelética fue medido a través de preguntas que investigaban la presencia o ausencia de dolor del miembro superior, columna, y miembro inferior con una data no mayor de siete días al momento de la evaluación; y presencia o ausencia de lesión del miembro superior, columna o miembro inferior. Las variables sobre los datos asociados a estilos de vida, como consumo de alcohol y hábito tabáquico, presencia de diabetes o hipertensión arterial, y las variables sociodemográficas como edad y género fueron recolectadas mediante el uso de cuestionarios validados en población nacional $^{25}$. El auto-reporte de calidad de sueño se midió con el cuestionario Sleep Self-Report, el cual determina que a mayor puntaje, peor calidad de sueño, y se dicotomizó según la media del puntaje obtenido en la muestra en baja calidad de sueño, y moderada/alta calidad de sueño ${ }^{26}$. Para la variable zona geográfica se agruparon las ciudades: Arica e Iquique como zona norte; Viña del Mar, Santiago y Talca como zona centro; y Los Ángeles, Osorno y Puerto Montt como zona sur. El peso corporal y la estatura bípeda fueron auto-reportadas ${ }^{27,28}$. El estado nutricional se calculó a partir del índice de masa corporal (IMC), el cual fue obtenido dividiendo el peso corporal por la estatura bípeda al cuadrado $\left(\mathrm{kg} / \mathrm{m}^{2}\right)$ y se clasificó en base a las recomendaciones de la Organización Mundial de la Salud (OMS) ${ }^{29}$. El auto-reporte de restricción de la movilidad por cuarentena/confinamiento debido a la pandemia por COVID-19 fue medido en los estudiantes a través de un cuestionario y se clasificó como 0,1 a 4, y mayor o igual a 5 semanas de confinamiento o cuarentena, ya sea voluntaria u obligatoria ${ }^{30}$.

\section{Análisis estadístico}

Los datos fueron analizados con el software SPSS 23.0 (SPSS 23.0 para Windows, SPSS Inc., IL, USA) y se consideró un alfa de 0,05 para todos los análisis. Las variables son presentadas como promedios o proporciones con intervalos de confianza a $95 \%(95 \% \mathrm{CI})$ para variables continuas y categóricas, respectivamente. Una prueba $\mathrm{t}$ de Student para muestras independientes se utilizó para establecer posibles diferencias en las variables continuas. Se utilizó la prueba Exacta de Fisher para establecer posibles asociaciones entre funcionalidad del miembro superior y variables independientes categóricas y un análisis de regresión logística binaria (método de entrada) ajustado por variables independientes de interés: nivel de actividad física y percepción de la condición física, antecedentes musculoesqueléticos (dolor y lesión de miembro superior, inferior, y columna), antecedentes sociodemográficos, estilos de vida, y situación aislamiento COVID-19 (semanas en cuarentena). Los resultados fueron presentados como Odds Ratio (OR) y sus respectivos 95\% IC.

\section{Resultados}

En la Tabla 1 se presentan las características sociodemográficas, antecedentes de COVID-19 y estilos de vida de la muestra según funcionalidad basado en el cuestionario DASH. Por su parte, los antecedentes musculoesqueléticos, nivel de actividad física y percepción de la condición física según funcionalidad, se observan en la Tabla 2. En resumen, la mayoría de los participantes presentaron una funcionalidad conservada $(92,1 \%)$ $\mathrm{y}$ en menor proporción una funcionalidad disminuida $(7,9 \%)$. La mayor prevalencia de funcionalidad disminuida del miembro superior se observó en participantes de sexo femenino $(79,6 \%)$, con una edad igual o mayor a 21 años $(61,2 \%)$, cuarentena por un período igual o mayor a 5 semanas $(81,6 \%)$, dolor de miembro 
Tabla 1, Características sociodemográficas, antecedentes de COVID-19 y estilos de vida de la muestra según \% de estudiantes que presentan y no presentan funcionalidad disminuida de miembro superior. Resultados basados en el cuestionario Discapacidades del Brazo, Hombro y Mano (DASH)

\begin{tabular}{|c|c|c|c|c|c|c|c|}
\hline \multirow{2}{*}{\begin{tabular}{|l} 
Antecedentes \\
Sociodemográficos
\end{tabular}} & \multicolumn{3}{|c|}{$\begin{array}{l}\text { Funcionalidad conservada } \\
\qquad \text { IC } 95 \%\end{array}$} & \multicolumn{3}{|c|}{$\begin{array}{l}\text { Funcionalidad disminuida } \\
\text { IC } 95 \%\end{array}$} & \multirow[t]{2}{*}{ Valor p* } \\
\hline & & & & & & & \\
\hline Participantes (\%) & 92,1 & 89,7 & 94,0 & 7,9 & 6,0 & 10,3 & $<0,001$ \\
\hline Edad (media en años) & 22,7 & 22,5 & 23,0 & 21,7 & 20,8 & 22,6 & 0,845 \\
\hline $\begin{array}{l}\text { Categoría Edad (\%) } \\
<21 \text { años } \\
\geq 21 \text { años }\end{array}$ & $\begin{array}{l}22,9 \\
77,1\end{array}$ & $\begin{array}{l}19,4 \\
73,6\end{array}$ & $\begin{array}{l}26,4 \\
80,6\end{array}$ & $\begin{array}{l}38,8 \\
61,2\end{array}$ & $\begin{array}{l}24,6 \\
47,1\end{array}$ & $\begin{array}{l}52,9 \\
75,4\end{array}$ & 0,013 \\
\hline $\begin{array}{l}\text { Sexo }(\%) \\
\text { Femenino } \\
\text { Masculino }\end{array}$ & $\begin{array}{l}54,9 \\
45,1\end{array}$ & $\begin{array}{l}50,8 \\
41,0\end{array}$ & $\begin{array}{l}59,0 \\
49,2\end{array}$ & $\begin{array}{l}79,6 \\
20,4\end{array}$ & $\begin{array}{r}67,9 \\
8,7\end{array}$ & $\begin{array}{l}91,3 \\
32,1\end{array}$ & 0,001 \\
\hline $\begin{array}{l}\text { Antropométricos (med } \\
\text { Estatura }(\mathrm{m}) \\
\text { Masa corporal }(\mathrm{kg}) \\
\text { IMC }\left(\mathrm{kg} / \mathrm{m}^{2}\right)\end{array}$ & $\begin{array}{l}1,67 \\
70,8 \\
25,2\end{array}$ & $\begin{array}{l}1,67 \\
69,6 \\
24,8\end{array}$ & $\begin{array}{l}1,68 \\
72,0 \\
25,5\end{array}$ & $\begin{array}{l}1,63 \\
66,0 \\
24,6\end{array}$ & $\begin{array}{l}1,61 \\
61,6 \\
23,2\end{array}$ & $\begin{array}{l}1,66 \\
70,4 \\
26,0\end{array}$ & $\begin{array}{l}0,897 \\
0,004 \\
0,013\end{array}$ \\
\hline $\begin{array}{l}\text { Estado nutricional (\%) } \\
\text { Bajo peso } \\
\text { Normopeso } \\
\text { Sobrepeso } \\
\text { Obeso }\end{array}$ & $\begin{array}{r}2,3 \\
52,4 \\
34,8 \\
10,5\end{array}$ & $\begin{array}{r}1,3 \\
48,4 \\
31,0 \\
8,2\end{array}$ & $\begin{array}{r}3,8 \\
56,5 \\
38,8 \\
13,3\end{array}$ & $\begin{array}{r}12,2 \\
38,8 \\
40,8 \\
8,2\end{array}$ & $\begin{array}{r}5,7 \\
26,4 \\
28,2 \\
3,2\end{array}$ & $\begin{array}{l}24,2 \\
52,8 \\
54,8 \\
19,2\end{array}$ & 0,001 \\
\hline $\begin{array}{l}\text { Zona geográfica (\%) } \\
\text { Norte } \\
\text { Centro } \\
\text { Sur }\end{array}$ & $\begin{array}{l}20,3 \\
41,3 \\
38,5\end{array}$ & $\begin{array}{l}17,2 \\
37,3 \\
34,6\end{array}$ & $\begin{array}{l}23,8 \\
45,3 \\
42,5\end{array}$ & $\begin{array}{l}22,4 \\
40,8 \\
36,7\end{array}$ & $\begin{array}{l}13,0 \\
28,2 \\
24,7\end{array}$ & $\begin{array}{l}35,9 \\
54,8 \\
50,7\end{array}$ & 0,930 \\
\hline $\begin{array}{l}\text { Pandemia COVID-19 } \\
\text { Semanas en cuarenten } \\
0 \text { semana } \\
1 \text { a } 4 \text { semanas } \\
\geq 5 \text { semanas }\end{array}$ & $\begin{array}{l}21,9 \\
16,6 \\
61,5\end{array}$ & $\begin{array}{l}18,7 \\
13,8 \\
57,5\end{array}$ & $\begin{array}{l}25,4 \\
19,9 \\
65,4\end{array}$ & $\begin{array}{r}2,0 \\
16,3 \\
81,6\end{array}$ & $\begin{array}{r}0,1 \\
8,5 \\
68,6\end{array}$ & $\begin{array}{l}10,7 \\
29,0 \\
90,0\end{array}$ & 0,003 \\
\hline $\begin{array}{l}\text { Estilos de vida } \\
\text { Hábito tabáquico (\%) } \\
\text { No-fumador } \\
\text { Fumador }\end{array}$ & $\begin{array}{l}77,3 \\
22,7\end{array}$ & $\begin{array}{l}73,7 \\
19,5\end{array}$ & $\begin{array}{l}80,5 \\
26,3\end{array}$ & $\begin{array}{l}77,6 \\
22,4\end{array}$ & $\begin{array}{l}64,1 \\
13,0\end{array}$ & $\begin{array}{l}87,0 \\
35,9\end{array}$ & 0,563 \\
\hline $\begin{array}{l}\text { Consumo de alcohol ( } \\
\text { No-consume } \\
\text { Consume }\end{array}$ & $\begin{array}{l}47,2 \\
52,8\end{array}$ & $\begin{array}{l}43,1 \\
48,7\end{array}$ & $\begin{array}{l}51,3 \\
56,9\end{array}$ & $\begin{array}{l}55,1 \\
44,9\end{array}$ & $\begin{array}{l}41,3 \\
31,9\end{array}$ & $\begin{array}{l}68,1 \\
58,7\end{array}$ & 0,181 \\
\hline $\begin{array}{l}\text { Diabetes mellitus tipo } \\
\text { No } \\
\text { Si }\end{array}$ & $\begin{array}{r}96,9 \\
3,1\end{array}$ & $\begin{array}{r}95,1 \\
2,0\end{array}$ & $\begin{array}{r}98,0 \\
4,9\end{array}$ & $\begin{array}{l}89,8 \\
10,2\end{array}$ & $\begin{array}{r}78,2 \\
4,4\end{array}$ & $\begin{array}{l}95,6 \\
21,8\end{array}$ & 0,028 \\
\hline $\begin{array}{l}\text { Hipertensión arterial (a } \\
\text { No } \\
\text { Si }\end{array}$ & $\begin{array}{r}97,0 \\
3,0\end{array}$ & $\begin{array}{r}95,3 \\
1,9\end{array}$ & $\begin{array}{r}98,1 \\
4,7\end{array}$ & $\begin{array}{r}91,8 \\
8,2\end{array}$ & $\begin{array}{r}80,8 \\
3,2\end{array}$ & $\begin{array}{l}96,8 \\
19,2\end{array}$ & 0,075 \\
\hline $\begin{array}{l}\text { Calidad del sueño (\%) } \\
\text { Baja } \\
\text { Moderada/alta }\end{array}$ & $\begin{array}{l}50,0 \\
50,0\end{array}$ & $\begin{array}{l}45,9 \\
45,9\end{array}$ & $\begin{array}{l}54,1 \\
54,1\end{array}$ & $\begin{array}{l}73,5 \\
26,5\end{array}$ & $\begin{array}{l}59,7 \\
16,2\end{array}$ & $\begin{array}{l}83,8 \\
40,3\end{array}$ & $<0,001$ \\
\hline
\end{tabular}

*Prueba Exacta de Fisher (variables categóricas) y t de Student (variables continuas). IC: intervalo de confianza; IMC: índice de masa corporal. 
Tabla 2. Antecedentes musculoesqueléticos, nivel de actividad física y condición física de la muestra según $\%$ de estudiantes que presentan y no presentan funcionalidad disminuida de miembro superior. Resultados basados en el cuestionario Discapacidades del Brazo, Hombro y Mano (DASH)

\begin{tabular}{|c|c|c|c|c|c|c|c|}
\hline \multirow{2}{*}{$\begin{array}{l}\text { Antecedentes } \\
\text { Musculoesqueléticos }\end{array}$} & \multicolumn{3}{|c|}{$\begin{array}{l}\text { Funcionalidad conservada } \\
\qquad \text { IC } 95 \%\end{array}$} & \multicolumn{3}{|c|}{$\begin{array}{l}\text { Funcionalidad disminuida } \\
\qquad \text { IC } 95 \%\end{array}$} & \multirow[t]{2}{*}{$\begin{array}{c}\text { Valor } \\
\text { p* }\end{array}$} \\
\hline & & & & & & & \\
\hline \multicolumn{8}{|c|}{ Dolor miembro superior (\%) } \\
\hline No & 63,3 & 59,3 & 67,1 & 8,2 & 3,2 & 19,2 & \multirow[t]{2}{*}{$<0,001$} \\
\hline Sí & 36,7 & 32,9 & 40,7 & 91,8 & 80,8 & 96,8 & \\
\hline \multicolumn{8}{|l|}{ Dolor columna (\%) } \\
\hline No & 54,0 & 49,9 & 58,1 & 34,7 & 22,9 & 48,7 & \multirow[t]{2}{*}{0,007} \\
\hline Sí & 46,0 & 41,9 & 50,1 & 65,3 & 51,3 & 77,1 & \\
\hline \multicolumn{8}{|c|}{ Dolor miembro inferior (\%) } \\
\hline No & 67,3 & 63,4 & 71,0 & 40,8 & 28,2 & 54,8 & \multirow[t]{2}{*}{$<0,001$} \\
\hline Sí & 32,7 & 29,0 & 36,6 & 59,2 & 45,2 & 71,8 & \\
\hline \multicolumn{8}{|c|}{ Lesión miembro superior (\%) } \\
\hline No & 61,5 & 57,5 & 65,4 & 20,4 & 11,5 & 33,6 & \multirow[t]{2}{*}{$<0,001$} \\
\hline Sí & 38,5 & 34,6 & 42,5 & 79,6 & 66,4 & 88,5 & \\
\hline \multicolumn{8}{|c|}{ Lesión columna (\%) } \\
\hline No & 49,5 & 45,4 & 53,6 & 42,9 & 30,0 & 56,7 & \multirow[t]{2}{*}{0,230} \\
\hline Sí & 50,5 & 46,4 & 54,6 & 57,1 & 43,3 & 70,0 & \\
\hline \multicolumn{8}{|c|}{ Lesión miembro inferior (\%) } \\
\hline No & 84,1 & 80,9 & 86,9 & 65,3 & 51,3 & 77,1 & \multirow[t]{2}{*}{0,002} \\
\hline Sí & 15,9 & 13,1 & 19,1 & 34,7 & 22,9 & 48,7 & \\
\hline \multicolumn{8}{|c|}{ Nivel de actividad física y percepción de la condición física (\%) } \\
\hline \multicolumn{8}{|c|}{ Nivel de actividad física (\%) } \\
\hline Baja & 37,6 & 33,7 & 41,6 & 40,8 & 28,2 & 54,8 & \multirow[t]{2}{*}{0,381} \\
\hline Moderada/alta & 62,4 & 58,4 & 66,3 & 59,2 & 45,2 & 71,8 & \\
\hline \multicolumn{8}{|c|}{ Condición física general } \\
\hline Baja & 22,2 & 18,9 & 26,0 & 32,7 & 21,2 & 46,6 & \multirow[t]{2}{*}{0,349} \\
\hline Moderada/alta & 77,8 & 74,0 & 81,1 & 67,3 & 53,4 & 78,8 & \\
\hline \multicolumn{8}{|c|}{ Condición cardiorrespiratoria } \\
\hline Baja & 28,3 & 24,8 & 32,1 & 38,8 & 26,4 & 52,8 & \multirow[t]{2}{*}{0,086} \\
\hline Moderada/alta & 71,7 & 67,9 & 75,2 & 61,2 & 47,2 & 73,6 & \\
\hline \multicolumn{8}{|c|}{ Condición muscular } \\
\hline Baja & 64,9 & 60,9 & 68,7 & 77,6 & 64,1 & 87,0 & \multirow[t]{2}{*}{0,047} \\
\hline Moderada/alta & 35,1 & 31,3 & 39,1 & 22,4 & 13,0 & 35,9 & \\
\hline \multicolumn{8}{|l|}{ Velocidad-agilidad } \\
\hline Baja & 25,0 & 21,6 & 28,7 & 38,8 & 26,4 & 52,8 & 0,029 \\
\hline Moderada/alta & 75,0 & 71,3 & 78,4 & 61,2 & 47,2 & 73,6 & \\
\hline Flexibilidad & & & & & & & \\
\hline Baja & 32,3 & 28,6 & 36,3 & 40,8 & 28,2 & 54,8 & 0,147 \\
\hline Moderada/alta & 67,7 & 63,7 & 71,4 & 59,2 & 45,2 & 71,8 & \\
\hline
\end{tabular}

*Prueba Exacta de Fisher (variables categóricas) y t de Student (variables continuas). IC, intervalo de confianza; IMC, índice de masa corporal. 
superior $(91,8 \%)$, lesión de miembro superior (79,6\%), y autopercepción baja de la condición muscular $(77,6 \%)$. Cabe destacar que en esta misma categoría de funcionalidad se observó un $12,2 \%$ de participantes con un estado nutricional de bajo peso.

En la Figura 1 se pueden observar los factores asociados a la disminución de la funcionalidad del miembro superior en estudiantes universitarios en situación de pandemia por COVID-19. En este contexto, se pudo identificar que los participantes con dolor $(\mathrm{OR}=17,60$; $95 \%$ IC $=5,16$ a 59,99 ; $p=0,001)$ y lesión $(\mathrm{OR}=10,43 ; 95 \% \mathrm{IC}=3,85 \mathrm{a}$ $28,27 ; p=0,001)$ del miembro superior presentaron la mayor probabilidad de disminución de la funcionalidad del miembro superior. Además, un estado nutricional de bajo peso $(\mathrm{OR}=10,48 ; 95 \%$ $\mathrm{IC}=2,21$ a 49,$60 ; p=0,003)$, cuarentena por $5 \mathrm{o}$ más semanas $(\mathrm{OR}=9,11 ; 95 \% \mathrm{IC}=1,08$ a 77,20 ; $p=0,043)$ y lesión de columna $(\mathrm{OR}=2,67 ; 95 \%$ $\mathrm{IC}=1,07$ a 6,$71 ; p=0,036)$ son factores de riesgo que se asocian a la disminución de la funcionalidad del miembro superior. Por el contrario, los participantes de sexo masculino (OR $=0,19 ; 95 \%$ IC $=0,07$ a 0,$53 ; p=0,001)$ y aquellos con una condición muscular moderada/alta $(\mathrm{OR}=0,34$; $95 \%$ IC $=0,13$ a 0,$91 ; p=0,031)$ presentaron una menor probabilidad de disminución de la funcionalidad del miembro superior.

\section{Discusión}

Los principales resultados de este estudio señalan que tener antecedentes de dolor y lesión musculoesquelética en miembro superior, antecedentes de lesión de columna, mantener un estado nutricional de bajo peso, y además, encontrarse con restricción de la movilidad por cuarentena mayor o igual a cinco semanas, se asocian a mayor probabilidad de presentar disminución de funcionalidad en miembro superior. Mientras que pertenecer al género masculino, y mantener una percepción de condición muscular moderada a alta se asocian a baja probabilidad. Estos resultados podrían ser de utilidad para la implementación de programas de vigilancia y promoción en la población universitaria, la cual, como se ha expuesto en estudios anteriores, presenta una alta prevalencia conducta sedentaria, baja condicion fisica, varias horas dedicadas al estudio, conductas alimentarias inadecuadas, y antecedentes de algunas patologías crónicas asociadas a los estilos de vida, lo cual le expone a empeorar su calidad de vida durante su formación académica ${ }^{6,7,25}$.

Estos hallazgos concuerdan con reportes anteriores. Por ejemplo, se han identificado como factores predictores de la baja condición física en estudiantes universitarios, el mantener una conducta sedentaria, tener exceso de peso, presentar antecedentes de lesión musculoesquelética, y destinar una mayor cantidad de horas al estudio ${ }^{7}$. Por otro lado, también los hombres universitarios han mostrado tener menos probabilidades de tener bajos niveles de actividad y condición física que las mujeres ${ }^{7,31,32}$. Estos antecedentes son similares a los observados en nuestro estudio, donde se observó que los hombres mantenían una percepción de mejor condición muscular. Además, se determinó que $20,4 \%$ de los hombres y 79,6\% de las mujeres presentaron una funcionalidad disminuida del miembro superior, transformando de este modo al género, en un posible factor de riesgo de funcionalidad disminuida en miembro superior.

Por otra parte, algunos estudios también han encontrado prevalencias de dolor y discapacidad en extremidades superiores en estudiantes universitarios cercanos a $24 \%$, considerando como factores de riesgo el uso de smartphones, computadores, y la presencia de antecedentes musculoesqueléticos, tal como se observa en nuestra investigación ${ }^{33}$. En este sentido, reportes anteriores han establecido la presencia de trastornos musculoesqueléticos como un factor asociado a la discapacidad de miembros superiores ${ }^{13,14,33}$. Además, en el presente estudio se observó una mayor incidencia de diminución de la funcionalidad del miembro superior en estudiantes universitarios $(12,2 \%)$ con un estado nutricional de bajo peso y sexo femenino. Esta situación difiere de otros estudios y en poblaciones con otros rangos etarios, donde se asocia el sobrepeso y la obesidad a una mayor pérdida de funcionalidad o discapacidad por trastornos musculoesqueléticos degenerativos como la osteoartrosis en la edad adulta ${ }^{31,34}$. Posiblemente, el estado nutricional deficiente de los estudiantes universitarios puede estar relacionado con una disminución de la funcionalidad, ya que durante la época de rendimiento académico se ha demostrado un comportamiento alimentario poco saludable y una ingesta inadecuada de nutrientes ${ }^{31,32}$. Los estudiantes universitarios 


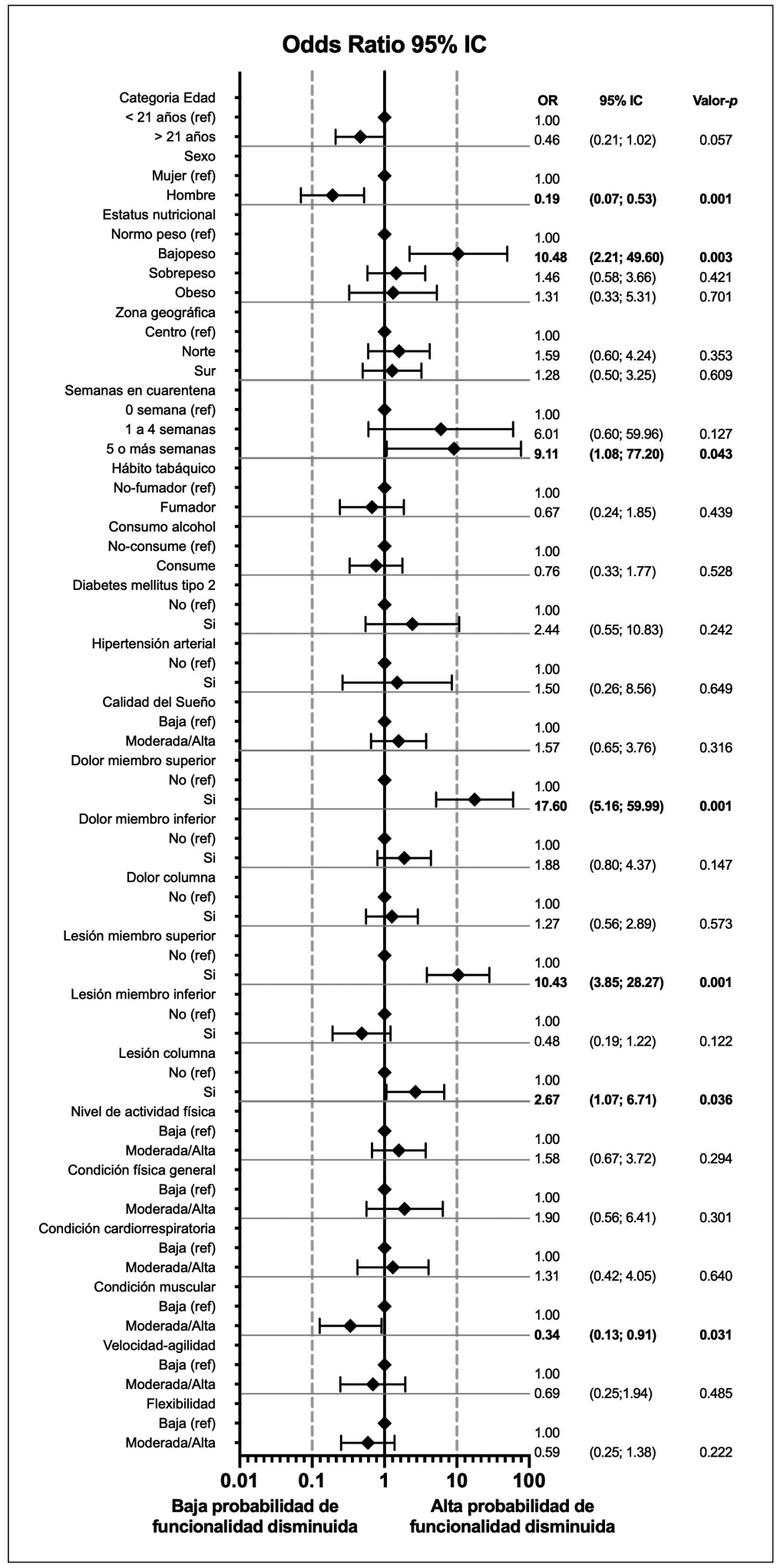

Figura 1. Factores asociados a la disminución de la funcionalidad del miembro superior en estudiantes universitarios en situación de pandemia por COVID-19. Odds ratio fue estimado mediante regresión logística binaria, $y$ asociaciones significativas fueron consideradas con un valor $\mathrm{p}<0,05$. 
habitualmente no desayunan, y ayunan por largas horas durante el día ${ }^{31,32}$, lo que sumado a una baja condicion fisica y el mantenimiento de varias horas sentado frente a las pantallas ${ }^{11,13,14}$, podría tener un efecto perjudicial en su condición muscular y postura corporal, causando disminución de la funcionalidad a largo plazo en sus miembros superiores ${ }^{11-14,33}$.

Por otra parte, mantenerse en situación de restricción de movilidad mayor o igual a 5 semanas producto de la pandemia por COVID-19, es un factor que se adiciona a los varios factores de riesgo mencionados anteriormente, ya que expone a los estudiantes a otros componentes, tales como el uso prolongado, masivo y unimanual de los smartphones ${ }^{33}$, necesidad de compatibilizar distintos esfuerzos en tareas domésticas junto con las académicas ${ }^{33}$, problemas en sus hábitos de sueño ${ }^{35}$, y presencia de estrés académico ${ }^{36}$, los cuales se han identificado como factores de riesgo para desarrollar trastornos musculoesqueléticos en extremidad superior ${ }^{33,36}$.

Existen algunas limitaciones en el presente estudio. Debido a la situación de pandemia por COVID-19, se usaron instrumentos de auto-reporte, lo cual podría ocasionar un sesgo por la infra o sobrevaloración de los participantes en relación con las variables estudiadas ${ }^{37}$. Sin embargo, el uso de cuestionarios validados y mediciones auto-reportadas, como peso y talla, han demostrado ser métodos aceptables y satisfactorios para su uso en estudios de vigilancia epidemiológica ${ }^{27,28}$.

En conclusión, pertenecer al género femenino, tener antecedentes de dolor y lesión musculoesquelética en miembro superior, antecedentes de lesión de columna, mantener un estado nutricional de bajo peso, encontrarse con restricción de la movilidad por cuarentena mayor o igual a cinco semanas, y tener una baja percepción de la condición muscular se asocian a mayor probabilidad de presentar disminución de funcionalidad en miembro superior. La identificación temprana de estos factores de riesgo modificables pueden ayudar a implementar acciones preventivas y/o rehabilitadoras en el hogar y/o instituciones de educación superior.

Agradecimientos: Se agradece de manera especial al equipo profesional del Núcleo de Investigación Interdisciplinaria en Salud NIIS, quienes desarrollaron y aplicaron los instrumentos a nivel nacional a los estudiantes. Todos los autores revisaron el manuscrito y están de acuerdo con su versión final. Ninguno de los autores reporta tener conflictos de intereses.

\section{Referencias}

1. Organización Mundial de la Salud (OMS). Prevención y control de infecciones en los centros de atención de larga estancia en el contexto de la COVID-19: orientaciones provisionales, 21 marzo de 2020 [Internet]. Disponible en: https://apps.who.int/iris/bitstream/ handle/10665/331643/WHO-2019-nCoV-IPC_long_ term_care-2020.1-spa.pdf? sequence $=1$ \&isAllowed $=y$ [consultado el 7 de agosto de 2020].

2. Pacheco-Barrios K, Cardenas-Rojas A, Giannoni-Luza S, Fregni F. COVID-19 pandemic and Farr's law: A global comparison and prediction of outbreak acceleration and deceleration rates. PLoS ONE 2020; 15 (9): e0239175. doi: 10.1371/journal.pone.0239175.

3. Aurenque D. Consideraciones éticas en contexto de triage por COVID-19 [Ethical considerations in the context of triage by COVID-19]. Rev Med Chile 2020; 148 (4): 562-63. doi: 10.4067/s0034-98872020000400562.

4. Mera AY, Tabares-González E, Montoya-González S, Muñoz-Rodríguez DI, Monsalve Vélez, F. Recomendaciones prácticas para evitar el desacondicionamiento físico durante el confinamiento por pandemia asociada a COVID-19. Universidad y Salud 2020; 22(2): 166-77. doi: 10.22267/rus.202202.188.

5. Chen P, Mao L, Nassis GP, Harmer P, Ainsworth BE, Li F. Coronavirus disease (COVID-19): The need to maintain regular physical activity while taking precautions. Journal of Sport and Health Science 2020; 9(2): 103-4. doi: 10.1016/j.jshs.2020.02.001.

6. Concha-Cisternas Y, Guzmán-Muñoz E, Valdes-Badilla P, Lira-Cea C, Petermann F, Celis-Morales C. Factores de riesgo asociados a bajo nivel de actividad física y exceso de peso corporal en estudiantes universitarios. Rev Med Chile 2018; 146(8):840-9. doi: 10.4067/s003498872018000800840.

7. Petermann F, Durán E, Labraña AM, Martínez MA, Leiva AM, Garrido-Méndez A, et al. Factores asociados al desarrollo de obesidad en Chile: resultados de la Encuesta Nacional de Salud 2009-2010 [Risk factors for obesity: analysis of the 2009-2010 Chilean health survey]. Rev Med Chile 2017; 145(6): 716-22. doi: 10.4067/ s0034-98872017000600716.

8. Concha-Cisternas Y, Castillo-Retamal M, GuzmánMuñoz E. Comparación de la calidad de vida en estu- 
diantes universitarios según nivel de actividad fisica. Revista Univ Salud 2019; 22(1): 33-40. doi: 10.22267/ rus.202201.172.

9. Pantic I, Malbasa M, Ristic S, Turjacanin D, Medenica $S$, Paunovic J, et al. Screen viewing, body mass index, cigarette smoking and sleep duration in Belgrade University student population: results of an observational, cross-sectional study. Rev Med Chile 2011; 139(7): 896901. doi: 10.4067/S0034-98872011000700010.

10. Garrote-Rojas D; Jiménez-Fernández S; Gómez-Barreto I. Problemas Derivados del Uso de Internet y el Teléfono Móvil en Estudiantes Universitarios. Form Univ 2018; 11(2): 99-108. doi: 10.4067/S0718-50062018000200099.

11. Harris C, Straker L, Pollock, C. \& Trinidad, S. Musculo-skeletal outcomes in children using information technology - the need for a specific etiological model. International Journal of Industrial Ergonomics 2005; $35,131-138$.

12. Dixon D, Johnston M, McQueen M, Court-Brown C. The Disabilities of the Arm, Shoulder and Hand Questionnaire (DASH) can measure the impairment, activity limitations and participation restriction constructs from the International Classification of Functioning, Disability and Health (ICF). BMC Musculoskelet Disord 2008; 9: 114. doi:10.1186/1471-2474-9-114

13. Casas A, Patiño M. Prevalencia y factores asociados con el dolor de espalda y cuello en estudiantes universitarios. Salud UIS 2012; 44(2): 45-55.

14. Fonseca M, Moraga A. Desórdenes del sistema musculoesquelético por trauma acumulativo en estudiantes universitarios de computación e informática. Ciencia y Tecnología 2010; 26(1 y 2): 1-18.

15. Straker L, Briggs A, Greig A. The effect of individually adjusted workstations on upper quadrant posture and muscle activity in school children. Work 2002; 18(3): 239-48.

16. Stodden D, Langendorfer S, Roberton MA. The association between motor skill competence and physical fitness in young adults. Res Q Exerc Sport 2009; 80(2): 223-9. doi: 10.1080/02701367.2009.10599556.

17. Godoy A, Valdés P, Fariña C, et al. [Association Between Fitness, Nutritional Status And Academic Performance In Physical Education Students]. Nutr Hosp 2015; 32(4): 1722-28. doi: 10.3305/nh.2015.32.4.9592.

18. World Health Organization (WHO). Global action plan for the prevention and control of noncommunicable diseases 2013-2020. Geneva, Switzerland: World Health Organization, 2013. Disponible en: http://apps.who.int/ iris/bitstream/10665/94384/1/9789241506236_eng.pdf.

19. Hudak PL, Amadio PC, Bombardier C. Development of an upper extremity outcome measure: the DASH (disa- bilities of the arm, shoulder and hand) [corrected]. The Upper Extremity Collaborative Group (UECG). Am J Ind Med 1996; 29(6): 602-8. doi: 10.1002/(SICI)1097-02 74(199606)29:6<602::AID-AJIM4>3.0.CO;2-L. Erratum in: Am J Ind Med 1996 Sep;30(3):372.

20. Angst F, Schwyzer H, Aeschlimann A, Simmen B, Goldhahn J. Measures of adult shoulder function: Disabilities of the Arm, Shoulder, and Hand Questionnaire (DASH) and its short version (Quick DASH), Shoulder Pain and Disability Index (SPADI), American Shoulder and Elbow Surgeons (ASES) Society standardized shoulder assessment form, Constant (Murley) Score (CS), Simple Shoulder test (SST), Oxford Shoulder Score (OSS), Shoulder Disability Questionnaire (SDQ), and Western Ontario Shoulder Instability Index (WOSI). Arthritis Care Res (Hoboken) 2011; 63 Suppl 11: S174-88. doi: 10.1002/acr.20630.

21. Hunsaker F, Cioffi D, Amadio P, Wright J, Caughlin B. The American academy of orthopaedic surgeons outcomes instruments: normative values from the general population. J Bone Joint Surg Am 2002; 84(2): 208-15. doi: 10.2106/00004623-200202000-00007.

22. Vinas BR, Barba LR, Ngo J, Majem LS. Validity of the international physical activity questionnaire in the Catalan population. Gac Sanit 2013; 27(3): 254-7. doi: 10.1016/j.gaceta.2012.05.013.

23. Serón P, Muñoz S, Lanas F. Nivel de actividad física medida a través del cuestionario internacional de actividad física en población chilena. Rev Med Chile 2010; 138(10): 1232-39. doi: 10.4067/S003498872010001100004 .

24. Ortega FB, Sánchez-López M, Solera-Martínez M, Fernández-Sánchez A, Sjöström M, Martínez-Vizcaino V. Self-reported and measured cardiorespiratory fitness similarly predict cardiovascular disease risk in young adults. Scand J Med Sci Sports 2013; 23: 749-57. doi: 10.1111/j.1600-0838.2012.01454.x.

25. Ministerio de Salud (MINSAL). Encuesta Nacional de Salud ENS 2009-2010. Chile: Ministerio de Salud, 2010. Disponible en: http://web.minsal.cl/portal/url/item/ bcb03d7bc28b64dfe040010165012d23.pdf.

26. Orgilés M, Owens J, Espada JP, Piqueras JA, Carballo JL. Spanish version of the Sleep Self Report (SSR): factorial structure and psychometric properties. Child Care Health Dev 2013; 39: 288-295. doi: 10.1111/j.13652214.2012.01389.x.

27. Dekkers JC, van Wier MF, Hendriksen IJM, Twisk JWR, van Mechelen W. Accuracy of self-reported body weight, height and waist circumference in a Dutch overweight working population. Bmc Med Res Methodol 2008; 8: 69. doi: 10.1186/1471-2288-8-69. 
28. Yoong SL, Carey ML, D'Este C, Sanson-Fisher RW. Agreement between self-reported and measured weight and height collected in general practice patients: a prospective study. Bmc Med Res Methodol 2013; 13: 38. doi: 10.1186/1471-2288-13-38.

29. World Health Organization (WHO). Obesity: Preventing and managing the Global Epidemic. 2000. Disponible en: http://www.who.int/nutrition/publications/ obesity/WHO_TRS_894/en/

30. Sánchez-Villena AR, de La Fuente-Figuerola V. COVID-19: cuarentena, aislamiento, distanciamiento social y confinamiento, ¿son lo mismo?, Anales de Pediatría 2020; 93(1): 73-4. doi: 10.1016/j.anpedi.2020.05.001.

31. Ratner GR, Hernández JP, Martel AJ, Atalah SE. Calidad De La Alimentación Y Estado Nutricional En Estudiantes Universitarios de 11 Regiones De Chile. Rev Med Chile 2012; 140: 1571-79. doi: 10.4067/S003498872012001200008.

32. Rodríguez RF, Palma LX, Romo BA, Escobar BD, Aragú GB, Espinoza OL, et al. Hábitos alimentarios, actividad física y nivel socioeconómico en estudiantes universitarios de Chile. Nutr Hosp 2013; 28(2): 447-55. doi: 10.3305/nh.2013.28.2.6230.
33. Almomani F, Alghwiri AA, Alghadir AH, Al-momani A, Iqbal A. Prevalence of upper limb pain and disability and its correlates with demographic and personal factors. J Pain Res 2019; 12: 2691-2700. doi: 10.2147/JPR. S198480.

34. World Health Organization (WHO). Overweight and obesity. Geneva, Switzerland: World Health Organisation; 2020. Disponible en: http://www.who.int/mediacentre/factsheets/fs311/en/

35. Bigalke JA, Greenlund IM, Carter JR. Sex differences in self-report anxiety and sleep quality during COVID-19 stay-at-home orders. Biol Sex Differ 2020; 11(1): 56. doi: 10.1186/s13293-020-00333-4.

36. Vranceanu AM, Safren S, Zhao M, Cowan J, Ring D. Disability and psychologic distress in patients with nonspecific and specific arm pain. Clin Orthop Relat Res 2008; 466(11): 2820-6. doi: 10.1007/s11999-008-0378-1.

37. Durán S, Crovetto M, Espinoza V, Mena F, Oñate G, Fernández M, et al. Caracterización del estado nutricional, hábitos alimentarios y estilos de vida de estudiantes universitarios chilenos: estudio multicéntrico. Rev Med Chile 2017; 145(11): 1403-11. doi: 10.4067/s003498872017001101403. 